\title{
Optical Remote Sensing Techniques in Biological Oceanography
}

\section{W Paul Bissett', Oscar Schofield ${ }^{2}$, Curtis D Mobley ${ }^{3}$, Michael F Crowley and Mark A Moline ${ }^{4}$}

'Florida Environmental Research Institute, Tampa, Florida, USA; ${ }^{2}$ Coastal Ocean Observation Laboratory, Institute of Marine and Coastal Sciences, Rutgers University, New Brunswick, New Jersey, USA; ${ }^{3}$ Sequoia Scientific, Inc., Redmond, Washington, USA; ${ }^{4}$ Biological Sciences Department, California Polytechnic State University, San Luis Obispo, California, USA

remote sensor $\mathrm{n}$. any instrument, such as a radar device or camera, that scans the earth or another planet from space in order to collect data about some aspect of it. - remote-sensing adj., n. (Collins English Dictionary)

\section{INTRODUCTION}

Light from the sun is the driving energy source behind all of the surface water biological processes. The radiant energy is harvested and stored as chemical energy through the process of photosynthesis providing the organic fuel for most of the oceanic food web. Single-cell marine phytoplankton are responsible for the majority of this energy conversion, and the growth of their organic biomass via autotrophic photosynthesis is referred to as primary production (Parsons et al., 1984). Oceanic net primary production is about one-third of the global net primary production (Denman et al., 1996). The estimate of oceanic biomass and net primary production has been revised upwards over the last two decades. 
This revision occurred in part because of the data stream provided by the first ocean color satellite sensor, the Coastal Zone Color Scanner (CZCS), and the scientific efforts of the NIMBUS-7 Experiment Team (NET) and many other ocean color scientists (Acker, 1994).

As visible light enters the water column, the in situ constituents, including water itself, impact the light's directionality and color. In pure seawater, blue light $(\sim 430 \mathrm{~nm})$ is least impacted by the processes of absorption and scattering. Exact measurements of absorption and scattering of pure water are extremely difficult to make. The actual pure absorption minima may be closer to $418 \mathrm{~nm}$ (Pope and Fry, 1997). However, scattering by water molecules decreases as wavelength increases (Smith and Baker, 1981), which leads to a transparency minima near $430 \mathrm{~nm}$. Most phytoplankton have evolved to efficiently utilize this region of the spectrum to maximize their photosynthetic activities (Kirk, 1994; Falkowski and Raven, 1997). In the presence of sufficient light and macro- (e.g. nitrogen and phosphorous) and micro-nutrients (e.g. iron), phytoplankton growth can lead to increases in total autotrophic biomass and organic degradational products. As the total organic load increases, the amount of absorptive and scattering material increases, reducing the total photon density as well as altering the spectral nature of that density, i.e. the color of the water shifts from the blue towards the red and the water clarity is reduced. The shift in hue as a function of water column biomass has been one of the more useful relationships that have been exploited for remote sensing purposes. By examining the shift in relative terms, i.e. dividing the upwelling light from the blue region by the upwelling light in the green region, a quantitative empirical relationship between 'color' and phytoplankton biomass was found in open ocean waters (Gordon et al., 1983; Gordon, 1987; Gordon et al., 1988; Mueller and Austin, 1992). These types of relationships have been used with the CZCS data to produce the first large-scale synoptic estimates of phytoplankton biomass. This type of relationship continues to be used today with the more recent ocean color sensor (Plate 5), Sea Wide Field-of-view Sensor [SeaWiFS].

The absorption of light by phytoplankton results primarily from the light-harvesting pigments within the thylakoid membrane, as well as photoprotective pigments found in the chloroplast envelope. Chlorophyll $a$ is the ubiquitous pigment found in all marine algae (Rowan, 1989), and as such has been used as a proxy for total phytoplankton biomass. The use of this pigment as a proxy for autotrophic biomass has been criticized because of the extreme variances in the ratio of chlorophyll $a$ per cell (Buck et al., 1996; Stramski et al., 1999). However, the techniques for measuring chlorophyll $a$ are relatively simple (Yentsch and Menzel, 1963; Holm-Hansen and Riemann, 1978; Bissett et al., 1997) and there are numerous empirical relationships between total chlorophyll $a$ and phytoplankton standing stock, as well as total primary productivity. Thus, this pigment has been used for decades as the measure of phytoplankton biomass. Usage of a pigment as an indicator of biomass was also heuristically appealing to ocean color scientists because of the direct link between pigments and absorption of light in the water column. 
This chapter will describe the basics of ocean color remote sensing. It will include a description of how to obtain and use SeaWiFS data within NASA's freely available ocean color remote sensing software. In addition, we will describe some differences in methodology and touch upon some of the more recent developments in the optical remote sensing field.

\section{PRINCIPLE}

\section{Geometrical radiometry}

Our discussion starts ${ }^{2}$ with a short review of radiometry, geometry, and radiative transfer theory. Optical remote sensing is concerned with the measurement of radiant energy (light) after a target or medium of interest has modified it. Light is defined in terms of energy units of joules $(\mathrm{J}=1 \mathrm{~kg}$ $\left.\mathrm{m}^{2} \mathrm{~s}^{-2}\right)$, or power units of watts $\left(\mathrm{W}=1 \mathrm{~J} \mathrm{~s}^{-1}\right)$. Alternatively, we could speak of light as individual packets called photons or quanta (wave-particle duality is a cornerstone of modern physics (Mobley, 1994)). An einstein is equal to a mol of photons ( 1 einst $=6.023 \times 10^{23}$ photons; a more recently accepted nomenclature is $1 \mathrm{~mol}$ quanta $=1$ einst). These definitions of light are related by the wavelength, the speed of light, and Planck's constant:

$$
q=\frac{h c}{\lambda}
$$

where $q$ is equal to the energy of a photon; $h$ is Planck's constant $=6.626 \times$ $10^{-34} \mathrm{~J} \mathrm{~s}$; $c$ is the speed of light $=2.998 \times 10^{8} \mathrm{~m} \mathrm{~s}^{-1}$; and $\lambda$ is the wavelength (in meters; note that $c$ is given in $\mathrm{m} \mathrm{s}^{-1}$; usage of this formula requires that wavelength and speed of light have the correct units) of interest.

The most useful measurements of light for remote-sensing purposes are radiance $(L)$ and irradiance $(E)$. Radiance is operationally defined as:

$$
L=\frac{\Delta Q}{\Delta t \Delta A \Delta \Omega \Delta \lambda}\left(\mathrm{J} \mathrm{s}^{-1} \mathrm{~m}^{-2} \mathrm{sr}^{-1} \mathrm{~nm}^{-1}\right)
$$

which states that radiance is the amount of energy $\Delta Q$, received in a time interval $\Delta t$, by a detector of area $\Delta A$, which is viewing a solid angle $\Delta \Omega$, and whose wavelength filter passes a wavelength band of size $\Delta \lambda$. The measurement of a solid angle is given in steradians (sr). It refers to the area of a sphere subtended by a set of radi from the sphere's center divided by the radius of the sphere squared. The best way to visualize the concept of a solid angle is to imagine yourself inside a sphere, at its center, holding an empty paper towel tube to your eye. Your eye can see an area on the surface of the sphere, through the tube, of size AREA. The distance from the center of the sphere to the surface of the sphere is the RADIUS, thus the solid angle $\Omega=$ AREA/RADIUS ${ }^{2}$ in steradians. As the total area of the sphere is $4 \pi$ (radius) $^{2}$, the solid angle of an entire sphere is equal to $4 \pi(\mathrm{sr})$. 
By this analogy, the remote-sensing instrument is essentially a collecting tube (the empty paper towel tube of the above example) with a detector at its base (your eye). The inside of the tube is painted black to minimize photons coming from outside the desired solid angle from bouncing off the inside sides of the tube into the detector. A diffuser is typically placed before the detector, so that the detector only has to sample a fraction of the area of the diffuser to determine the total incoming radiance. The surface area of the diffusing plate has an area, $A$, associated with it, such that all of the terms of Equation (2) are now defined. Figure 26.1 gives a schematic drawing of such an instrument.

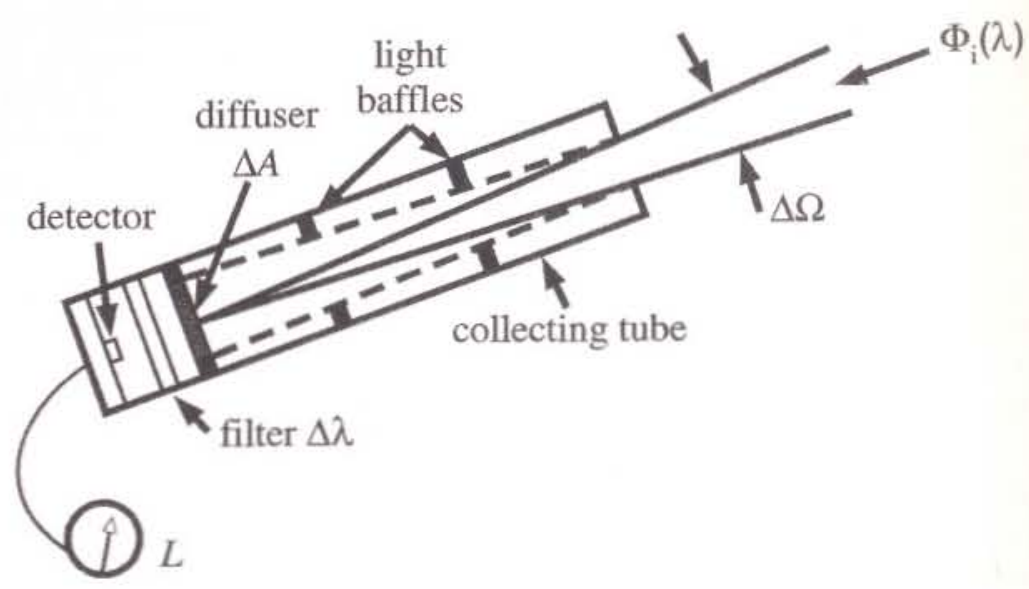

Figure 26.1. Schematic design of an instrument for measuring unpolarized spectral radiance (redrawn from Mobley (1994)).

As we are talking about the pointing of collecting tubes, we need to understand a couple of terms about directionality. A sensor looking straight down is said to have a viewing angle, or nadir angle, of $0^{\circ}$. As the sensor moves 'off' nadir, this angle changes in a positive direction, such that a horizontal view would be $90^{\circ}$, and a vertically (upward) looking sensor would have a nadir angle of $180^{\circ}$. As the sensor moves off vertical viewing, it acquires an azimuthal viewing angle, $\phi$, which is typically measured clockwise from the instrument's (satellite) direction of travel.

Ocean color sensors are called passive sensors, which means they do not have an illuminating, or active, source of light, but rather, passively collect the light coming from the planet. In order to quantify the information derived from light impacting a passive detector flying high above the earth, we first need to know what the total irradiance at the area of interest on the surface of the planet is. Thus, the other useful measurement for remote sensing is downwelling irradiance. If we remove the tube from our above instrument, and set it on the ground facing upward, it would collect light from all downward directions. The integration of all downwardtraveling photons over all nadir and azimuthal angles is called down- 
welling irradiance, $E_{\mathrm{d}}$. The detector, however, does not see the radiance equally over all solid angles. Consider a laser looking straight down onto the detector (nadir angle, $\theta$, of 0 degrees), whose beam exactly fits onto the detector. Now consider the same laser at a $45^{\circ}$ angle to the detector. The beam will be spread out upon the ground, and the detector sees only a fraction of the total off-nadir light as it is dispersed over a larger projected area on the ground. The dispersal of the photon density is proportional to $\triangle A \cos \theta$. (The quantity $\triangle A \cos \theta$ is the area that the detector projects into the plane perpendicular to the beam direction). Thus, downwelling irradiance is simply the integral of radiance over all nadir and azimuthal angles multiplied by the cosine of the nadir angle.

\section{Why is the sky blue?}

We continue our discussion with the spectrum of sunlight and the impacts of a fluid medium (the atmosphere) on the downwelling light field. The visible solar irradiance at the top of the atmosphere is blue-rich (peaking in magnitude at $\sim 450 \mathrm{~nm}$ ). This irradiance from the sun is reduced as it passes through the atmosphere, and blue light is preferentially removed relative to red light in a clear atmosphere. The relative impact of the blue reduction becomes greater the more atmosphere the solar irradiance has to penetrate. This should be intuitively obvious for those who have seen the sun at noon and the sun at sunset. At noon the sun is directly overhead and the distance through the atmosphere is minimized, and it appears nearly white. At sunset, photons must pass through a greater volume of the atmosphere to arrive at the same point. The result is a sun that appears to be dimmer and shift in color towards red.

The reason for the color shift and reduction in energy has to do with the inherent and apparent optical properties (IOPs and AOPs) of the atmosphere. The inherent optical properties refer to the properties of a medium that impact a photon as it travels through a finite distance of the medium. These properties do not depend on the directionality of the photons. For this discussion we are going to assume there are only three possible processes that impact a photon as it passes into a given medium. First, the material in that medium can absorb the photon, completely removing it from the incoming radiant energy. Second, the photon can be scattered by the material, changing its directionality but otherwise not impacting the radiant power. Third, it can be transmitted through the medium without interaction at all. Let us define then three processes, absorptance $(A)$, scatterance $(B)$, and transmittance $(T)$ for a parallel beam of light traveling through some distance, $\Delta r$, of medium (Figure 26.2):

$$
A(\lambda)=\frac{\Phi_{a}(\lambda)}{\Phi_{i}(\lambda)}, \quad B(\lambda)=\frac{\Phi_{b}(\lambda)}{\Phi_{i}(\lambda)}, \quad T(\lambda)=\frac{\Phi_{t}(\lambda)}{\Phi_{i}(\lambda)}
$$

where $\Phi(\lambda)$ refers to the radiant power in watts (W) incident on the medium, and $\Phi_{a}, \Phi_{b}$, and $\Phi_{t}$, refer to the radiant power attributed to each of the processes affecting the photons through the medium. The use of $\lambda$ 


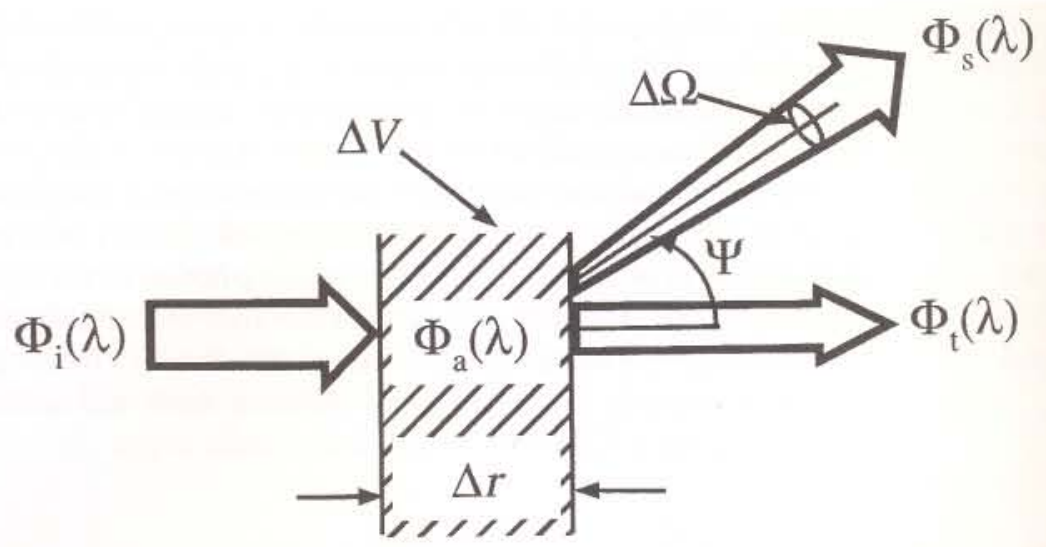

Figure 26.2. Geometry used to define inherent optical properties (redrawn from Mobley (1994)).

denotes the spectral dependence of each of the processes. (Note that while both absorptance and scatterance processes remove photons from the original direction of the incident beam, only absorptance truly removes the photons. Scatterance just changes the direction that they travel. However, this scattered light misses the detector placed in the path of the original beam.) We assume there are no changes in the radiant energy from internal sources of light within the medium and that no photons are absorbed and re-emitted at different wavelengths. Thus, $A(\lambda)+B(\lambda)+T(\lambda)=1$.

In our above example of the sun and atmosphere, the transmittance for blue light was less than that for green or red light. The matter in the atmosphere, i.e. oxygen, nitrogen, water vapor, clouds, dust, etc., absorb and scatter the photons traveling through it. This leads to a spectral shift in the radiant power of the total incoming light along the direct line of sight of the sun. If we are looking right at the sun, the process (absorptance or scatterance) that has the greatest impact on the reduction in the solar radiance is not completely obvious. However, if we look off angle (to the side) from the direct solar beam during a clear sky day, the dominant process removing blue light becomes obvious. As the sky is blue, we can infer that there must be some process that is preferentially removing blue light from the direct beam (scatterance), but is not completely removing the photons (absorptance). This process is called molecular scattering (often called Rayleigh scattering) and has a very strong wavelength dependence $\left(\lambda^{-1}\right)$.

The inherent optical properties of absorption and scattering, $a(\lambda)$ and $b(\lambda)$, respectively, are defined as the absorptance and scatterance per unit distance of medium, and are given in units of $\mathrm{m}^{-1}$. Beam attenuation, $c(\lambda)$, is equal to the sum of $a(\lambda)$ and $b(\lambda)$. The third inherent optical property that is important is the volume scattering function, $\beta(\psi, \lambda)$, and refers to both the change in directionality and reduction in incident radiant power through the solid angle $\Delta \Omega$ in Figure 26.2. Here, $\psi$ refers to the angle that the photon travels after being scattered by the medium. $\psi$ varies between 
$0^{\circ}$ (no change in direction) and $180^{\circ}$ (complete back scattering). Integration of the volume scattering function between angle $90^{\circ}$ and $180^{\circ}$ yields another important quantity called total backscattering coefficient, $b_{b}(\lambda)$, which has units of $\mathrm{m}^{-1}$.

As we mentioned above, the satellite sensor is a passive instrument. We now have the terminology to more rigorously describe what the sensor is detecting. An ocean color sensor measures the upwelling radiance that is derived from the incident solar irradiance which is backscattered in the field of view of our sensor?

\section{Biological considerations}

As stated above, phytoplankton have adapted their photosynthetic machinery to harvest light in the blue relative to the red. Figure 26.3 shows the absorption spectra for some major bloom-forming phytoplankton found in today's oceans. The spectra were measured for phytoplankton cultures in the laboratory but illustrate the variability in phytoplankton absorption due to differences in accessory pigments. Intuitively, the greater the phytoplankton concentration, the lower the total light available. In a purely absorbing medium the light is removed exponentially as a function of its absorption coefficient and the distance the photon has to travel, i.e.:

$$
L(z)=L(0) \exp (-a \cdot Z)
$$

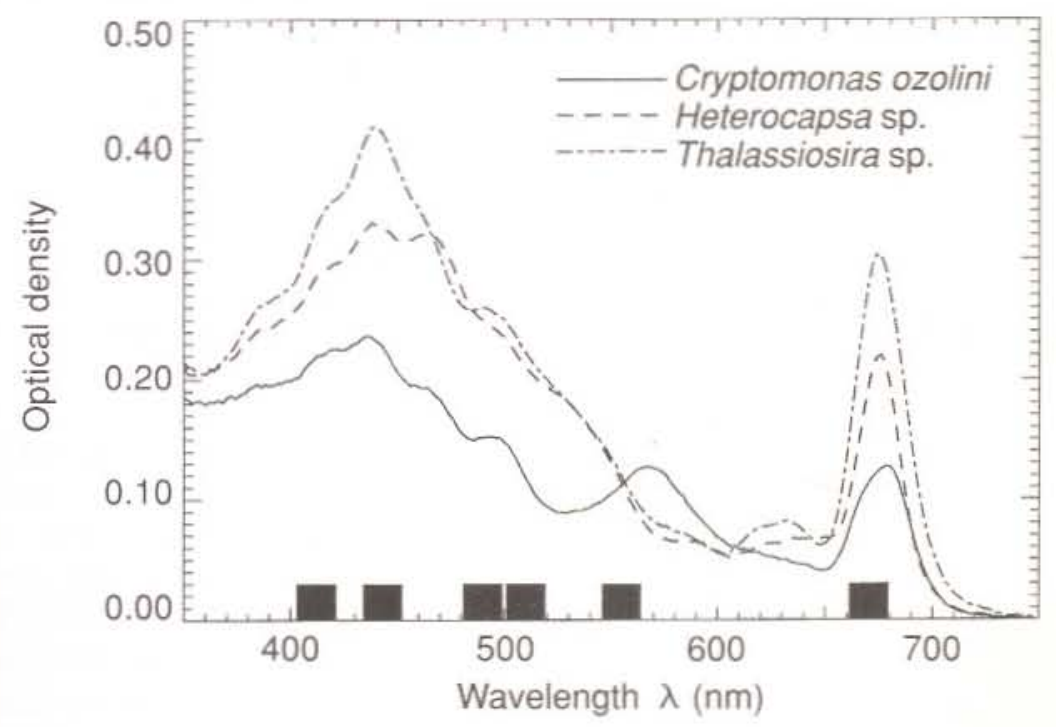

Figure 26.3. Optical density for three common phytoplankton species. The measurements were made in suspension on actively growing cultures held at Rutgers University. The suspensions were concentrated to $10.7 \times 10^{\circ}, 4.0 \times 10^{5}$, and $21.2 \times 10^{6}$ cells $\mathrm{ml}^{-1}$ for Cryptomonas ozolini, Heterocapsa sp. and Thalassiosira sp., respectively, and measured on a DW2 Aminco Spectrophotometer in split beam mode in a $1 \mathrm{~cm}$ cuvette. 
where $L(0)$ is the radiance at a boundary point; $L(z)$ is the radiance at a distance $z$ in a direct line from the boundary point; $a$ is the absorption coefficient in units $\mathrm{m}^{-1}$; and $\mathrm{Z}$ is the distance along the direct path. This is known as Lambert-Beer's law. What is evident from Equation (4) and Figure 26.3 is that the differences in absorption coefficients will manifest themselves exponentially in the water column. In other words, the preferential removal of blue light happens exponentially as phytoplankton concentration increases. Note the minimum in the absorption spectra in the area from $\sim 520$ to $600 \mathrm{~nm}$. With the exception of cyanobacteria, most phytoplankton species do not have pigment complements that strongly absorb light at these wavelengths. The net color effect of increasing the phytoplankton concentration is that the water will become increasingly green.

How green is green? And can a quantitative measure of 'greenness' be translated into an estimation of chlorophyll $a$ and/or other biological material? Using a rigorous radiative transfer code (HYDROLIGHT 4.0, http://www.sequoiasci.com/hydrolight.html) with a model of the water column IOPs as a function of chlorophyll $a$ concentrations in typical oceanic waters (Gordon and Morel, 1983; Morel, 1991), we computed the water-leaving radiance spectra, $L_{w}(\lambda)$, as the chlorophyll $a$ concentration increased from 0.10 to $10.0 \mathrm{mg} \mathrm{Chl} a \mathrm{~m}^{-3}$ (Figure 26.4(a)). Note the striking 'hinge point' near $490 \mathrm{~nm}$. By taking the ratio of $L_{w}(490)$ wavelengths to one of the green $L_{w v}(\lambda)$ on the right of the hinge, one could imagine that a non-linear relationship could be used to map the ratio of upwelling radiance to chlorophyll $a$ concentrations. This was the type of relationship used by the original Nimbus Experiment Team to formulate the empirical algorithm for the CZCS. The SeaWiFS algorithm (O'Reilly et al., 1998) follows the same format and is:

$$
\text { chl }=-0.040+10^{\left[0.341-3.001 X+2.811 X^{2}-2.041 X^{3}\right]}
$$

where

$$
X=\log 10\left[R_{\mathrm{rs}}(490) / R_{\mathrm{rs}}(555)\right]
$$

The SeaWiFS algorithm is a modification of the original CZCS-type algorithm as it uses remote-sensing reflectance, $R_{\mathrm{rs}}(490)$ and $R_{\mathrm{rs}}(555)$, rather than normalized $L_{w}(\lambda)$, in the empirical estimation of chlorophyll $a$ concentration (Gordon and Clark, 1981; Mueller and Austin, 1992). $R_{\mathrm{rs}}$ is defined as $L_{w}(\lambda) / E_{d}(\lambda)$. Normalizing by the downwelling light field, either by the Gordon and Clark (1981) method or by division by $E_{\mathrm{d}}(\lambda)$, removes the spectral variation and directionality of the source light from the upwelling radiance, i.e. relates all measurements 'to those that would be measured were the sun at the zenith, at the mean Earth-sun distance and with the effects of the atmosphere removed' (Mueller and Austin, 1992). Figure 26.4(b) shows the $R_{\mathrm{rs}}(\lambda)$ curves from the same HYDROLIGHT runs.

However, the process of absorption removes photons from the water. A satellite sensor does not view absorbed photons, rather it 'sees' the effects 

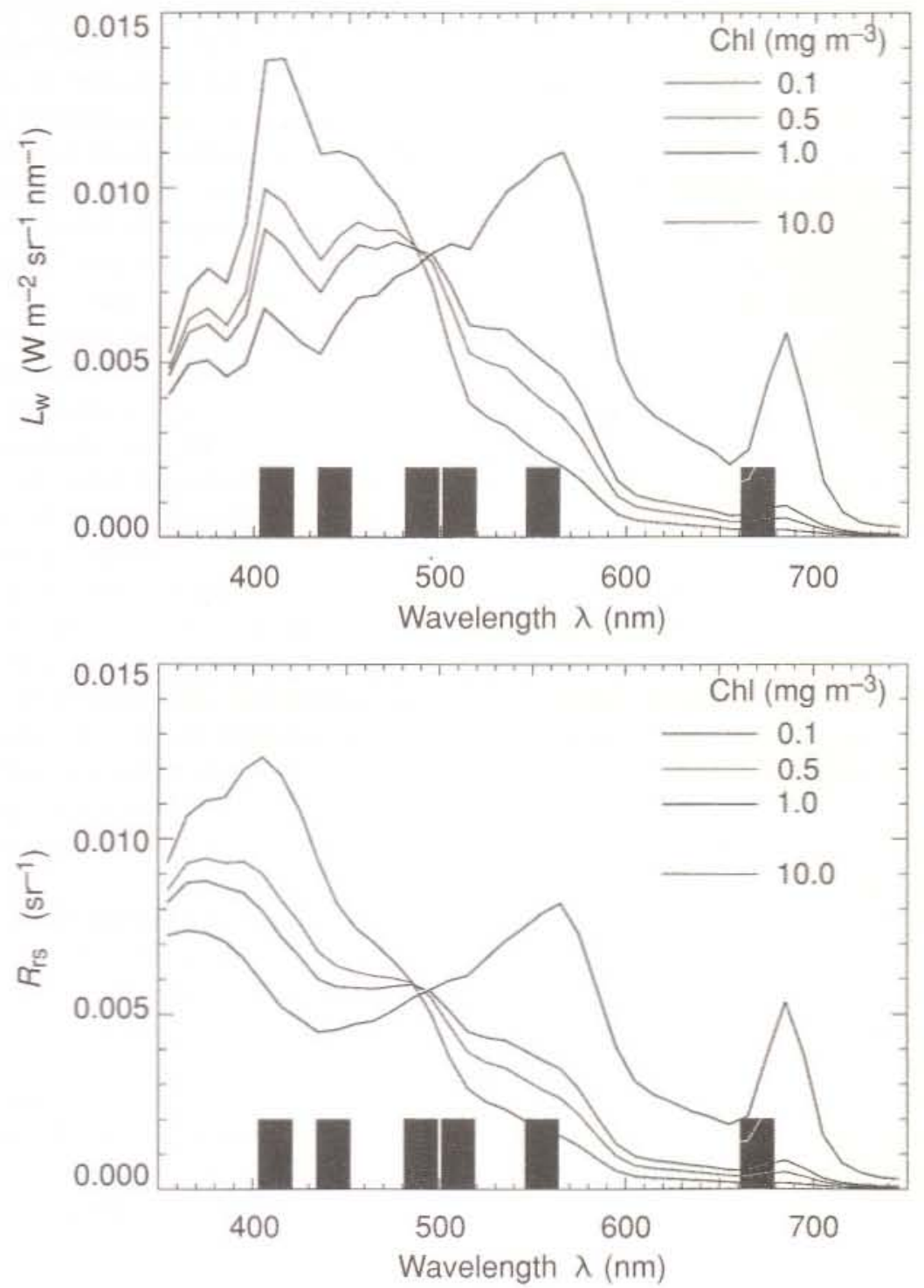

Figure 26.4. Modeled water-leaving radiance, $L_{w}(\lambda)$ at high and low chlorophyll $a$ concentrations. The water column IOPs were created with a bio-optical model for Case 1 waters (Gordon and Morel, 1983; Morel, 1991) as a chlorophyll a concentration. Note that the Case 1 model does not include non-covarying optical constituents, i.e. river CDOM or suspended sediments found in Case 2 waters. Near-shore Case 2 waters may have significantly different $L_{\text {, spectra for the same }}$ chlorophyll $a$ concentrations. The HYDROLIGHT runs used the Case 1 water IOP model, with the sun at $30^{\circ}$ nadir angle in a clear sky, wind of $5 \mathrm{~m} \mathrm{~s}^{-1}$, in infinitely deep water, Raman scattering and chlorophyll $a$ fluorescence (see the peaks around $680 \mathrm{~nm}$ ) were included in the runs. The width of the SeaWiFS data bands are shown as bars at the bottom of the figure. 
of absorption on the backscattered photons leaving the water. The decrease in $L_{w}$ (Figure 26.4) in the blue is remarkably similar to the increased blue absorption of phytoplankton (Figure 26.3). In fact, the AOP of $R_{\mathrm{rs}}$ (as well as the radiometric quantity of $L_{w}$ ) appears to be proportional to the IOP ratio of $b_{\mathrm{b}} / a$ (Morel and Prieur, 1977). The relationship established by Morel and Prieur (1977) links an IOP (absorption) to an AOP (remote sensing reflectance). One may expect a spectral dependence of $b_{\mathrm{b}}$. Fortunately, the spectra dependence of $b_{b}$ is less influenced by phytoplankton than the absorption coefficient because most of the backscattering comes from very small sub-micron size particles and water itself. Molecular scattering is nearly isotropic (equal in all directions), such that water molecules have a spectrally invariant backscattering ratio of $\sim 50 \%$. Viruses have a backscattering ratio of $\sim 20-30 \%$, increasing slightly in the red (Stramski and Mobley, 1997). As a general rule, the greater the size of the particle beyond the molecular size, the greater the scattering, but the lower the backscattering ratio. The size of the phytoplankton load does impact the total scattering coefficient, $b(\lambda)$, but the volume scattering function of phytoplankton is very weak in the backwards direction. The fraction of photons scattered backwards by phytoplankton ranges from about $\sim 0.01$ to $0.20 \%$ depending on the size and wavelength (increased scattering in the red). Over a range of typical phytoplankton concentrations (away from river plumes or areas of active sediment re-suspension) the variability of $b_{b}(\lambda) / a(\lambda)$ is mainly a function of the variability of $a(\lambda)$. The ratio of $L_{w}(490) / L_{w}(555)$ is thus nearly proportional to $a(555) / a(490)$ (or inversely proportional to $a(490) / a(555))$.

This relationship between the ratio of absorption (an IOP) and remote sensing reflectance (an AOP) is the basis for the current satellite algorithms to estimate chlorophyll $a$ concentration. There are many nuances to this relationship (the above relationship established by Morel and Prieur (1977) assumes that all the optical constituents co-vary with chlorophyll; this is obviously not true for vast sections of the coastal ocean), and the term 'nearly proportional' in the last sentence of the above paragraph is cause for great angst and research in the ocean color community. However, we have addressed the basics of ocean color remote sensing and will now focus the remainder of this chapter on the tools necessary to acquire and use satellite data from NASA. These tools will be demonstrated with actual images, and compared against in situ data so we can briefly discuss issues of validation.

\section{HARDWARE, SOFTWARE, AND DATA}

\section{Hardware requirements}

Image processing and analysis are computationally intensive processes. The tasks of navigation, atmospheric correction, re-mapping, and image manipulation typically require workstation caliber computers to accomplish. For our purposes, a workstation refers to a computer built around a 
RISC chip with a UNIX operating system, i.e. SGI O2 or SUN UltraSPARC. While the power of PC-type computers has dramatically increased in the past decade, the memory requirements and execution speed have yet to match those of the workstations. However, this is a rapidly changing field and the latest PC-type machines with the LINUX operating systems may yet prove to be sufficient for image processing 5 . NASA currently makes available a free software package to process SeaWiFS images as part of the Mission to Planet Earth [MTPE] program. This package is called SeaWiFS Data Analysis Software [SeaDAS], and it can be downloaded from http://seadas.gsfc.nasa.gov.

While there are other image processing packages available, our discussion will focus on SeaDAS, as it is free and can be used with a currently operational satellite sensor (SeaWiFS). There are many other ocean color satellites being planned (and one that has just been launched, i.e. MODIS), but the data streams are not currently available. The following discussions may become dated soon after publication, as the tools, techniques, and equipment are constantly changing. Our discussion should be viewed in the context of the process required to acquire an image from a public (NASA) image database, and used to understand some of the basics of retrieving and remapping satellite data to usable images. The reader is referred to a more complete text on remote sensing and image processing (Schowengerdt, 1997) for further information on techniques and algorithms.

On the NASA web site, one will find the suggested requirements for computational equipment. These will change as SeaDAS changes and the computational abilities of hardware and software change. While you may choose the minimum system requirements that NASA suggests, as a general rule, more is better than less in image processing. At the very least opt for more RAM and disk memory than the minimum requirements. The reason for the increase in memory is that when analyzing a time series of satellite images you may load and display multiple images at once, which will rapidly take up RAM. If you do not have sufficient RAM, most computers are set up to use disk memory as virtual RAM (also called SWAP memory). SeaDAS is written in Interactive Data Language [IDL], and our experience with this software suggests that is does not handle swapping very efficiently. The net result is that your system may freeze up, or your process may completely blow up, causing a loss of time and data, as well as endless frustration. The increase in disk space results from the fact that a single Level $1 \mathrm{~A}^{6}$ image and its Level $2^{7}$ processed products may be as large as $250 \mathrm{MB}$. This is without creating any data products using other algorithms, or creating publishable images.

\section{Software requirements}

The required software to run SeaDAS are:

- Operating systems: SGI:IRIX 6.3 or 6.5 , SUN: Solaris 2.6 or 2.7 , or Linux

- Required software: IDL 5.1 or 5.2 (from Research Systems, Inc.; http://www.rsinc.com) 
- Optional compiler: C, FORTRAN (required if users wish to compile SeaDAS from scratch)

- Software libraries: HDF 4.0r2 (included in SeaDAS)

SeaDAS does not require the full version of IDL and can be compiled solely with the runtime version of IDL. This may save some money on the initial start up of using SeaDAS. The disadvantage to using the runtime version is that you lose all of the functionality of IDL, which does have some powerful analytical tools.

\section{Data acquisition}

SeaWiFS data can be acquired from NASA's Distributed Active Archive Center [DAAC], and can be ordered online by following the ocean color links at http://daac.gsfc.nasa.gov/. SeaWiFS is a commercial instrument flying on Orbimage's (http://www.orbimage.com/) Orbview-2 spacecraft. NASA purchased data rights for its researchers prior to the satellite's launch. However, there are restrictions on how the data may be used. As long as you are doing non-profit research it is quite easy to become an Authorized SeaWiFS Data User. The links for the required documentation to become an Authorized Data User are on the Ocean Color page at the DAAC web site. Once you become an Authorized User, use the SeaWiFS data page (http://daac.gsfc.nasa.gov/data/dataset/SEAWIFS/index. html) to browse for the images you wish to acquire. New users must register here as well. Once you have registered with the DAAC, you are ready to acquire your images.

\section{APPLICATION}

Natural dynamics in microbial communities reflect biological responses to environmental fluctuations (variations to light, temperature, shear, and nutrients), trophic interactions, and physical transport processes such as turbulent mixing and advection. This has made characterizing the ecology of natural microbial communities difficult. Remote sensing provides a tool that can provide information over time/space scales not possible using traditional sampling approaches from ships (Plate 6). This has fundamentally changed our view of microbial dynamics of the oceans and provides the foundation for adaptive sampling of biological communities in the future (Schofield et al., 1999). Despite much promise, scientists should cautiously view the information provided by satellite maps.

We are going to demonstrate the power and pitfalls of ocean color data with an example from an active research program in the New York Bight [NYB]. Data were collected by the Coastal Ocean Observation Laboratory [COOL] at the Long-term Ecosystem Observatory [LEO-15], which is located off the central coast of New Jersey. The LEO-15 system is a coupled ocean observation/modeling system being constructed to acquire long-term high-resolution measurements from marine to coastal habitats (http://marine.rutgers.edu/cool). Currently the LEO-15 
observation network consists of satellites, aircraft, radar, meteorological sensors, subsurface observation nodes, moorings, research vessels and autonomous underwater vehicles. The system collects data from the Mullica River/Great Bay Estuarine Reserve and across the New York Bight. The data described here were collected as part of a study focused on summer upwelling.

We will now walk through the process of obtaining an image from the DAAC, processing the image, and then briefly analyzing the data in context of an ongoing coastal oceanographic program.

\section{Obtaining an image}

We are going to start by trying to obtain an image of the East Coast of the United States on July 16, 1999. Go to the DAAC web site (http://daac.gsfc.nasa.gov/data/dataset/SEAWIFS/index.html) and follow the links:

$\rightarrow$ Data Products

$\rightarrow$ LAC (local area coverage, $1 \mathrm{~km}$ resolution)

$\rightarrow$ L1A_HRPT (Level 1A data from the High Resolution Picture Transmission [HRPT] stations)

$\rightarrow$ HNSG (NASA Goddard Space Flight Center, Greenbelt, Maryland, USA HRPT station)

$\rightarrow 1999$ (data from the year 1999)

$\rightarrow$ July

$\rightarrow$ S1999197171620.L1A_HNSG

By clicking on this link, you will be shown a browse image of the HRPT data collected by the receiving station as the satellite was passing over Goddard. The browse image is an un-navigated pseudo-color image, which allows the viewer to see if the site of interest is in the scene and visible through the clouds. The image file name is from the time stamp on the image, i.e. day of year 197 (July 16th) of year 1999. Order the image by following the links at the top of the page, and be sure to request all of the meteorological data in the process.

The best way to receive the data is via FTP. When the data are ready you will receive an email with instructions on how to retrieve the data from the DAAC FTP server. The data will be available in compressed form, which you must uncompress on your computer after downloading it.

\section{Processing the image}

In the directory where you have uncompressed the image on your computer, start SeaDAS. This will place the SeaDAS Main Menu GUI on your desktop. On this interface choose:

$\rightarrow$ Process

$\rightarrow$ SeaWiFS

$\rightarrow 12$ gen (L2 file generation) 
On the L2 Products GUI you want to select the file that you ordered and uncompressed (S1999197171620.L1A_HNSG), and give a name for the output file, e.g. S1999197171620.L2. You can use all the parameter defaults. We would suggest using the meteorological and calibration files that came with the image in the MET file parameter block. Select the Run Button.

Once the image has finished processing, select the Quit Button and return to the SeaDAS Main Menu GUI interface. Now choose:

$\rightarrow$ Display

$\rightarrow$ seadisp (General image and graphics display)

This starts the Seadisp Main Menu GUI. Select:

$\rightarrow$ Load

$$
\rightarrow \text { SeaWiFS }
$$

which starts the Product Selection GUI. Select the file that you created with the 12 gen routine, and afterwards select the chlor_a check mark under the Products sub-page. This brings up the Band Selection GUI. You can display this product, however, it will not be mapped into a projection that is easy to use. Instead, on the SeaDisp Main Menu GUI select:

$\rightarrow$ Functions

$\rightarrow$ Projection

which starts the SeaDisp Projection GUI. Select the chlor_a band, and drop down to the Projections button and choose Cylindrical. Below this input panel, set the north and south latitude and east and west longitude coordinates for the desired limits of the image (Plate 6 limits are approximately $30.20^{\circ} \mathrm{N}$ and $30.75^{\circ} \mathrm{N}$, by $74.50^{\circ} \mathrm{W}$ and $73.75^{\circ} \mathrm{W}$ ), and click on the Go Button. A new band will be displayed in the Band List Selection GUI called Mapped -chloro_a (your filename). Display this image. A new window will be displayed with the mapped data. The Function Button will allow you to add coastlines, color palettes, output the data, etc.

\section{Basic image analysis}

The largest (non-seasonal) variations in ocean temperatures along the New Jersey coast are caused by episodic summertime upwelling events forced by southwesterly winds associated with the Bermuda High. Off the southern coast of New Jersey, topographic variations associated with ancient river deltas direct the upwelled water to evolve into an alongshore line of three recurrent upwelling centers that are co-located with historical regions of low dissolved oxygen [DO]. Remote sensing has been a key tool in mapping the cold, nutrient-rich, upwelled water. This nutrient-laden water supports large phytoplankton blooms when exposed to sunlight, which in turn provides a steady flux of organic material to the underlying bottom waters. Under the right conditions, the supply of organic material exceeds the supply of oxygenated waters, with subsequent remineralization leading to low oxygen conditions. The coherence between the cold 
upwelling water, as depicted by the sea surface temperature [SST] minimum (Plate 6(b)) near Node A, and the increase in SeaWiFS estimated chlorophyll $a$ concentration (Plate 6(a)) is clearly evident. This relationship between cold nutrient-rich water and sea surface pigments (when there is sufficient sunlight for autotrophic growth) in the NYB is an extension of the more general relationship, also depicted in Plate 5, over much of the world's oceans.

Use of remote sensing data places the LEO-15 field program in the context of the larger oceanic environment, providing the necessary information with which to view the in situ data (Plate 7). The in situ data of absorption and scattering collected along the transect line running to the north of Node A (Plate 6) confirms our theoretical interpretation of the effect of increasing biomass on the relative changes in ratios of $L_{w}(\lambda), a(\lambda)$, and $b_{b}(\lambda)$. As mentioned above (and seen in Equation (5)), we expect the ratio of $L_{w}(490) / L_{w}(555)$ to increase (seen as increasing chlorophyll $a$ in the SeaWiFS image) as the ratio of $a(490) / a(555)$ decreases, with very little spectral change in $b_{\mathrm{b}}(\lambda)$ (Figure 26.5; note that the difference in wavelength for $b_{b}(\lambda)$ is the result of the different available bands on the in situ instruments).

Plate 7 also demonstrates a source of error in the satellite-derived chlorophyll $a$ (and SST as well). The ocean is not a two-dimensional surface, but rather a three-dimensional fluid medium. However, satellites can only 'see' some small distance into the surface of the ocean. The depth at which information can be retrieved from remotely sensed data depends on the penetration depth of the light, which in turn is a function of the water column IOPs. Clearly, some wavelengths are going to penetrate deeper than others and the depth of penetration depends on how much 'stuff' is in the water. In homogeneous water, there is an exponential decay $^{9}$ in the photon density as light penetrates downward. Photons are backscattered into the upward direction at each depth, and there is also an

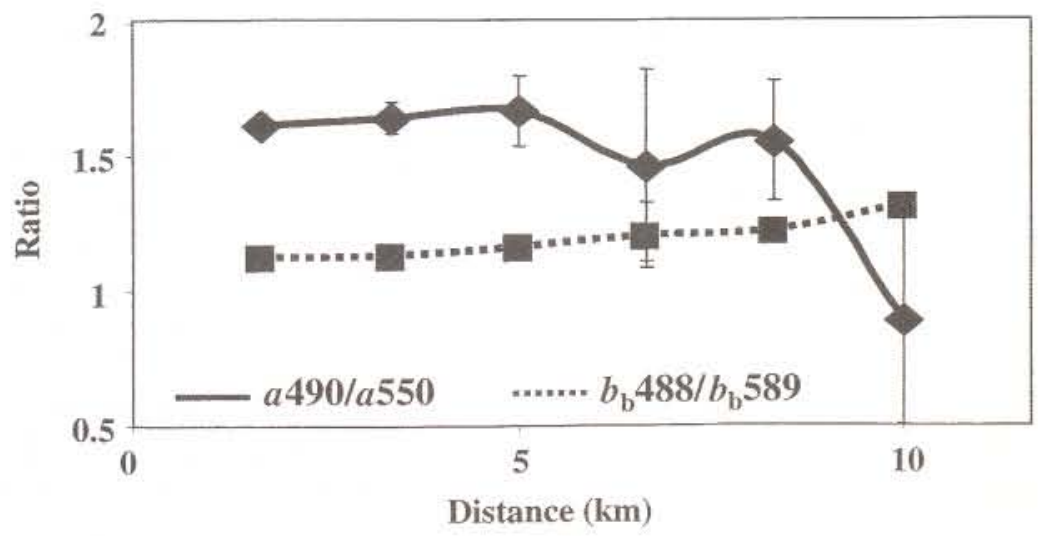

Figure 26.5. Ratio of the backscatter and absorption data for surface waters for the cross-shelf transect. While backscatter is relatively constant, the absorption ratio varied by over $50 \%$ reflecting changes in phytoplankton biomass. 
exponential decay in the photons traveling back toward the surface. This two-way travel by photons means that the information derived by the spectral change in water-leaving radiance is an integration of the water column's IOPs, heavily weighted by the near-surface values. Thus, any empirical approach relating in situ optical constituents to water-leaving radiance becomes an integrated estimate over the distance that the photons have penetrated, i.e. the chlorophyll $a$ estimates in Plate 6(a) are integrated near-surface values. The vertical dependence of the IOPs in Plate 7 is lost in the satellite data.

But how deep does the satellite 'surface' water extend? Based on the estimate of chlorophyll $a$, one could estimate the attenuation of downwelling irradiance, $K$, per unit distance and use an equation similar to Equation (4) to estimate the penetration depth of the light. In general, $\sim 90 \%$ of the light that leaves the surface waters come from the first diffuse attenuation depth (where diffuse attenuation depth is defined as $1 / \mathrm{K}$ ). Note that different colors of light integrate over different distances in the water because the diffuse attenuation $K$ depends strongly on wavelength. The additional complications owing to vertical variations of the IOPs have made it necessary to use empirical relationships like Equation (5) to estimate chlorophyll. This formulation will have wide error bars, but for the time being is a reasonable, all-purpose, algorithm for most open ocean conditions.

The algorithm in Equation (5) was primarily developed for open ocean conditions where the color signal is solely a function of the in situ produced organic material. In addition, it assumes that all of the organic colored constituents, i.e. phytoplankton, dead phytoplankton, phytodetritus, Colored Dissolved Organic Matter [CDOM], co-vary with each other. These kinds of waters are often referred to as Case 1 waters (Morel and Prieur, 1977), and they represent a majority of the world oceans ( $~ 80$ to $90 \%$ ). Coastal waters have additional sources of color that do not necessarily co-vary with primary production. The additional color constituents include re-suspended sediments, bottom reflection, river-derived CDOM, etc. Coastal waters that have non-covarying optical constituents are often referred to as Case 2 waters. For these more complicated coastal ocean conditions, new algorithms that specifically address the vertical structure of water column IOPs are being developed (Gould and Arnone, 1998). These usually require some additional information on in situ IOPs at the time of the image collection, which are subsequently used in conjunction with reflectance maps to derive three-dimensional IOP estimates.

In Plate 6, if we were to imagine a transect parallel to the $\mathrm{N}$ line through Node A, we would see chlorophyll $a$ decreasing with increasing temperature. This conforms to our general interpretation of organic material and water temperature (Plate 5). Northeast of Node A along the N transect line is a different story. The cold water seems to split an area of warmer water, such that transecting from the coast to deeper water yields a warm $\rightarrow$ cold $\rightarrow$ warm line. We would expect to see a commensurate low chlorophyll $\rightarrow$ high chlorophyll $\rightarrow$ low chlorophyll SeaWiFS plot. However, we see a much higher amount of estimated chlorophyll in water near the coast at temperatures near $20^{\circ} \mathrm{C}$ than we do offshore at similar temperatures. This 
higher chlorophyll estimate nearer to the shore results from optical constituents that do not co-vary with the chlorophyll $a$ concentration, i.e. reflectance of light off the bottom, higher concentrations of CDOM coming from the rivers and estuaries, and re-suspended sediments from the bottom. In Case 2 waters, great care must be taken in divining detailed information from simple algorithms and SeaWiFS data. Table 26.1 shows actual chlorophyll $a$ measurements along the $\mathrm{N}$ line in Plate 6 . Notice the increasing error in the SeaWiFS estimate as we move from offshore to onshore. The errors in the SeaWiFS estimates appear to stabilize around $\sim 5 \mathrm{mg}$ chl $a \mathrm{~m}^{-3}$ at approximately $10 \mathrm{~km}$ offshore. SeaWiFS estimated chlorophyll $a$ concentrations greater than this should be analyzed carefully in these types of waters.

Table 26.I Comparison between satellite-derived chlorophyll $a$ and in situ HPLC surface measurements of chlorophyll $a$. Sample corresponds to stations in Figure 26.5. The decreasing difference between in situ HPLC chlorophyll and SeaWiFS estimates results from the decreasing influence of non-covarying optical constituents, i.e. sediments, estuarine CDOM, etc., on the upwelling radiance signal.

\begin{tabular}{ccc}
\hline Distance from shore $(\mathrm{km})$ & SeaWiFS chl $a\left(\mathrm{mg} \mathrm{m}^{-3}\right)$ & HPLC chl $a\left(\mathrm{mg} \mathrm{m}^{-3}\right)$ \\
\hline 3.5 & 17.92 & 8.26 \\
6.5 & 8.86 & 6.80 \\
10.0 & 4.04 & 4.48 \\
\hline
\end{tabular}

\section{FUTURE DIRECTIONS}

There are many research efforts trying to develop more accurate IOP algorithms from ocean color data to address Case 2 water problems, as well as to derive the concentrations of other optically active constituents, e.g. CDOM. NASA's Sensor Intercomparison and Merger for Biological and Interdisciplinary Oceanic Studies (SIMBIOS; http://sim bios.gsfc.nasa.gov/) program is one of the mechanisms by which a large fraction of this work is funded. Their web site is a good starting point for the latest information on ocean color algorithm development.

There are other ocean color sensors, besides the SeaWiFS sensor, currently operating. These included the NASA Moderate Resolution Imaging Spectroradiometer (MODIS), the Indian Remote Sensing Satellite Modular Optoelectronic Scanner (MOS) and Ocean Color Monitor (OCM), the European POLarization and Directionality of the Earth's Reflectance (POLDER), and the Taiwanese Ocean Color Imager (OCI). The acquisition and manipulation of these data streams are a bit more difficult. However, more intensive studies into ocean color may find the usage of multiple remote sensing data streams a means to acquire more complete temporal and spatial coverage of a study site, as well as providing crosscalibration of the data streams. 


\section{CONCLUSIONS}

We have reviewed the basic tenets of ocean color remote sensing. By exploring how light penetrates the water column and how the optical constituents impact the light as it travels through the water, we hope to provide the basic understanding of the value and limitations of ocean color data. While we have shown where to obtain SeaWiFS images, and software to process these images, the reader should use this chapter as just one of many references on ocean color remote sensing. There is a large scientific difference between making color pictures and understanding the driving processes behind spatial variations of water-leaving radiance. Remote sensing is an indispensable tool in oceanography, and used correctly can greatly facilitative the interpretation of in situ and laboratory data.

\section{Acknowledgement}

This work was supported by the Office of Naval Research.

\section{Endnotes}

'Gross primary production minus plant respiration.

${ }^{2}$ While a complete description of radiometry and hydrological optics is beyond the scope of this chapter, there are some basic tenets of ocean optics that must be covered in order to proceed with the utilization of remote sensing data. For a more complete discussion of hydrological optics and its impacts on photosynthesis, see Mobley (1994) and Kirk (1994).

${ }^{3}$ Remember we have assumed that there are no internal sources of light or absorption/emission processes, e.g. solar stimulated fluorescence.

"The operational coefficients of this algorithm have changed. It should be noted that these coefficients frequently change, depending on new processing, atmospheric correction, etc., as well as region and seasonal changes for site- and timespecific studies.

${ }^{5}$ This is relative of course. Digital image processing has been a recognized field of endeavor for the last several decades, at the beginning of which supercomputers were but a fraction of the power of today's PCs. However, as the power of computers has increased, so have the demands of image processing, i.e. greater image size and resolution, more wavelength bands of information, etc.

'Level 1A [L1A]: reconstructed, unprocessed instrument data at full resolution, including radiometric and geometric calibration coefficients and georeferencing parameters (i.e. platform ephemeris) computed and appended, but not applied to the L0 data (see http://seadas.gsfc.nasa.gov/doc/sds_faq.html\#G_levels).

${ }^{7}$ Level [L2]: derived environmental variables at the same resolution and location as the $\mathrm{L} 1$ data.

${ }^{8}$ The next two sections are valid as of May 1, 2000. Future updates to the DAAC and SeaDAS software may render them obsolete. However, the processes of obtaining an image and deriving products will be similar, so the following descriptions may be used as a reference.

"Equation (4) with an attenuation term, $K$, instead of absorption, $a . K$ is a slight modification of $a$ resulting from the effects of backscattering and the average direction of the photon density. 


\section{References}

Acker, J. G. (1994). Volume 21, The Heritage of SeaWiFS: A Retrospective of the CZCS NIMBUS Experiment Team (NET) Program. NASA Center for AeroSpace Information: Linthicum Heights, MD.

Bissett, W. P., Patch, J. S., Carder, K. L. and Lee, Z. (1997). Pigment packaging and chlorophyll $a$-specific absorption in high-light oceanic waters. Limnol. Oceanogr. 42, 961-968.

Buck, K. R., Chavez, F. P. and Campbell, L. (1996). Basin-wide distributions of living carbon components and the inverted trophic pyramid of the central gyre of the North Atlantic Ocean, summer 1993. Aquatic Microbial Ecol. 10, 283-298.

Denman, K., Hofmann, E., Marchant, H., Abbott, M. R., Bates, T. S., Calvert, S. E., Fasham, M. J., Jahnke, R., Kempe, S., Lara, R. J., Law, C. S., Liss, P. S., Michaels, A. F., Pedersen, T. F., Peña, M. A., Platt, T., Sharp, J., Thomas, D. N., Scoy, K. A. V., Walsh, J. J. and Watson, A. J. (1996). Marine biotic responses to environmental change and feedbacks to climate. In: Climate Change 1995: The Science of Climate Change (J. T. Houghton, L. G. M. Filho, B. A. Callander, N. Harris, A. Kattenberg and K. Maskell, Eds), pp. 485-516. Cambridge, University Press, Cambridge.

Falkowski, P. G. and Raven, J. A. (1997). Aquatic Photosynthesis. Blackwell Sciences, Inc., Malden, MA.

Gordon, H. R. (1987). Bio-optical model describing the distribution of irradiance at the sea surface resulting from a point source embedded in the ocean. Appl. Opt. 26, 4133-4148.

Gordon, H. R. and Clark, D. K. (1981). Clear water radiances for atmospheric correction of coastal zone color scanner imagery. Appl. Opt. 20, 4175-4180.

Gordon, H. R. and Morel, A. (1983). Remote Assessment of Ocean Color for Interpretation of Satellite Visible Imagery, a Review. Springer-Verlag, New York.

Gordon, H. R., Brown, J. W., Brown, O. B., Evans, R. H. and Clark, D. K. (1983). Nimbus 7 CZCS; reduction of its radiometric sensitivity with time. Appl. Opt. 22, 3929-3931.

Gordon, H. R., Brown, O. B., Evans, R. H., Brown, J. W., Smith, R. C., Baker, K. S. and Clark, D. K. (1988). A semianalytic radiance model of ocean color. J. Geophys. Res. 93, 10,909-10,924.

Gould, R. W. and Arnone, R. A. (1998). Three-dimensional modelling of inherent optical properties in a coastal environment: coupling ocean colour imagery and in situ measurements. Int. J. Remote Sensing 19, 2141-2159.

Holm-Hansen, O. and Riemann, B. (1978). Chlorophyll a determination: improvements in methodology. OIKOS 30, 438-447.

Kirk, J. T. O. (1994), Light and Photosynthesis in Aquatic Ecosystems. Cambridge University Press, Cambridge.

Mobley, C. D. (1994). Light and Water. Academic Press, San Diego, CA.

Morel, A. (1991). Light and marine photosynthesis: a spectral model with geochemical and climatological implications. Prog. Oceanogr. 26, 263-306.

Morel, A. and Prieur, L. (1977). Analysis of variations in ocean color. Limnol. Oceanogr. 22, 709-722.

Mueller, J. L. and Austin, R. W. (1992). Volume 5, Ocean Optics Protocols for Sea WiFS Validation. NASA Center for AeroSpace Information, Greenbelt, MD.

O'Reilly, J. E., Maritorena, S., Mitchell, B. G., et al. (1998). Ocean color chlorophyll algorithms for SeaWiFs. J. Geophys, Res., 103, 24, 937-24, 953.

Parsons, T. R., Takahashi, M. and Hargrave, B. (1984). Biological Oceanographic Processes. Pergamon Press, New York.

Pope, R. M. and Fry, E. S. (1997). Absorption spectrum (380-700 nm) of pure water. II. Integrating cavity measurements. Appl. Opt. 36, 8710-8723. 
Rowan, K. S. (1989). Photosynthesis Pigments of Algae. Cambridge University Press, Cambridge.

Schofield, O., Grzymski, J., Bissett, W. P., Kirkpatrick, G., Millie, D. F., Moline, M. and Roesler, C. S. (1999). Optical monitoring and forecasting systems for harmful algal blooms: possibility or pipe dream? J. Phycol. 35, 1477-1496.

Schowengerdt, R. A. (1997). Remote Sensing; Models and Methods for Image Processing. Academic Press, San Diego, CA.

Smith, R. C. and Baker, K. S. (1981). Optical properties of the clearest natural waters (200-800 nm). Appl. Opt. 20, 177-184.

Stramski, D. and Mobley, C. D. (1997). Effects of microbial particles on oceanic optics: a database of single-particle optical properties. Limmol. Oceanogr. 42, 538-549.

Stramski, D., Reynolds, R. A., Kahru, M. and Mitchell, B. G. (1999). Estimation of particulate organic carbon in the ocean from satellite remote sensing. Science 285, 239-242.

Yentsch, C. S. and Menzel, D. W. (1963). A method for the determination of phytoplankton chlorophyll and phaeophytin by fluorescence. Deep-Sea Res. 10, 221-231. 